\title{
HISTOCHEMICAL DIFFERENCES IN FLIGHT AND LEG MUSCLES OF THE PIGEON
}

\author{
Xiang-Zhong Li ${ }^{1}$, Shunsuke KUMASA ${ }^{2}$, Masahiko ISOGAI ${ }^{2}$, \\ AND MASAHIKo MORI ${ }^{2 *}$ \\ Department of Cardiac Pathophysiology ${ }^{1}$, Institute of Basic Medical Sciences, \\ Harbin Medical University, People's Republic of China and \\ Department of Oral Surgery ${ }^{2}$, Asahi University School \\ of Dentistry, Hozumi, Motosu-gun, 501-02
}

Received for publication August 13, 1988, in revised form November 1, 1988 and re-revised form November 29, 1988

\begin{abstract}
Muscle fibers of flight (pectoralis major) and leg muscles (gastrocnemius) of the adult pigeon were classified based on succinate dehydrogenase (SDH) activity and myosin adenosine triphosphatase (ATPase) activity following preincubation at various pH's. The fiber structure in semithin as well as ultrathin sections was also evaluated. The pectoral muscle was composed almost entirely of the type IIB red fiber, with type IIB white fibers representing only $3.4 \%$ of the total fibers; and the average diameter of the type IIB red fibers was $26.8 \mu \mathrm{m} \pm 6.3$, whereas that of the type IIB white was $50.9 \mu \mathrm{m} \pm 7.9$. The pectoral muscle also contained a larger amount of fat droplets and mitochondria in the type IIB red fiber, while relatively smaller amounts of fat droplets and mitochondoria were found in the IIB white fiber. These findings suggest that the major part of pigeon pectoral muscle should be categorized as a fast-twitch (alkali-stable ATPase, Type IIB) and fatigue resistant (high oxidative staining). The leg muscle of pigeon was divided into three types, the type I fiber, the type IIA and IIB fiber. Fiber diameter of the three fiber types was not much different, and the fiber population was $23.0 \%$ type I, $33.3 \%$ type IIA and $43.6 \%$ type IIB. Regarding histochemical properties, the flight and leg muscles were very different in their muscle fibers, thus reflecting the different functions of the two muscles.
\end{abstract}

With the use of oxidative enzyme histochemical techniques for the detection of succinate dehydrogenase (SDH) and myosin adenosine triphosphatase (ATPase) activities, skeletal muscle fibers have been classified into two types; red and white or type I and type II (6). With preincubation at different $\mathrm{pH}$ employed $(\mathrm{pH} \mathrm{4.2,} \mathrm{4.6,} \mathrm{and}$ 9.4) in the detection of myosin ATPase activity it was possible to identify and discriminate 4 different types of skeletal muscle fibers (1). Typing of skeletal muscle fibers has also been distinguished from semithin sections as well as electronmicroscopic features $(12,14)$. Histochemical fiber typing have been reflected by their functional activities as fast and slow muscles or with fast-twitch and slow-twitch, and have been recognized with responsible involvement of neuromuscular disorders (4).

Pigeons as well as other avians have well developed flight muscles, especially the pectoralis major muscle. These features have been evaluated as having considerable overlap between the fiber types for metabolic parameters and structural function data 
$(3,5,13,16,17,19)$.

The present study was undertaken to distinguish fiber types in pectoral and gastrocnemius muscles respectively, in the pigeon and to compare them. Also, muscle fiber types were identified in semithin sections by simple staining with a modification of the p-phenylene-diamine (PPD) method.

\section{MATERIALS AND METHODS}

Pectoral major and gastrocnemius muscles were dissected from adult male pigeons. These two muscles were employed for the following 3 purposes: enzyme histochemistry, light microscopic observation of semithin sections, and electron microscopic observation of ultrathin sections.

For enzyme histochemistry, fresh materials were frozen in isopentane precooled to $-160^{\circ} \mathrm{C}$ by liquid nitrogen. Serial transverse sections were cut at $15 \mu \mathrm{m}$ in a $-20^{\circ} \mathrm{C}$ cryostat and air-dried for $30 \mathrm{~min}$. The sections were stained for $\mathrm{SDH}$ activity with Nitro-BT ditetrazolium chloride. For investigating the $\mathrm{pH}$ lability of myosin ATPase the modified method of Brooke and Kaiser (1) was employed. Type I, IIA and IIB fibers were identified according to the criteria of Brooke and Kaiser (1). Acid preincubation was performed for $5 \mathrm{~min}$ at $\mathrm{pH}$ ranging from 4.0 to $4.7 \mathrm{in} 0.1 \mathrm{pH}$-steps, in a barbital acetate buffer. Prior to incubation, the sections were rinsed for one min in $18 \mathrm{mM} \mathrm{CaCl}_{2}$ in $0.02 \mathrm{M}$ barbital buffer ( $\mathrm{pH} 9.4$ ). Alkaline preincubation consisted of $15 \mathrm{~min}$ at $\mathrm{pH} 10.0-10.9(0.1 \mathrm{pH}$ steps), in a medium composed of $18 \mathrm{mM}$ $\mathrm{CaCl}_{2}$ in a $25 \mathrm{mM} \mathrm{Na}$-barbital. All sections were then incubated for $45 \mathrm{~min}$ in a medium containing $18 \mathrm{mM} \mathrm{CaCl}_{2}$, in a $25 \mathrm{mM} \mathrm{Na-barbital} \mathrm{(pH} \mathrm{9.4)} \mathrm{and} 4.5 \mathrm{mM}$ ATP. All sections were maintained at room temperature.

The diameters of 400-500 fibers in the cryostat sections of each muscle were measured directly with a MAGISCAN 2A semiautomatic image analyzer (JoyceLoebl, England).

For semithin sections and ultrathin sections of the muscle specimens, muscles were cut into longitudinal strips of approximately $3 \mathrm{~mm}$ thickness. The pieces were fixed for two hr in $2 \%$ glutaraldehyde in $0.1 \mathrm{M}$ cacodylate buffer $(\mathrm{pH} \mathrm{7.2).} \mathrm{The}$ tissues were then rinsed in cacodylate buffer (containing 10\% sucrose), post-fixed in buffered osmium, dehydrated though a graded ethanol series, and embedded in Epon 812. Semithin sections $(1.0-1.5 \mu \mathrm{m})$ were cut on a Huxley Ultramicrotome with a wet knife, transferred to clean glass slides, and dried on a hot plate. The sections were stained by a modified p-phenylene-diamine (PPD) method employing double staining. The slides were first immersed in $0.05 \%$ basic fuchsin in $2.5 \%$ ethanol at $60^{\circ} \mathrm{C}$ for $3 \mathrm{~min}$. They were then rinsed in distilled water, air-dried, submerged in $1 \%$ p-phenylene-diamine solution dissolved in methanol-isopropanol alcohol $(\mathrm{PPD})(\mathrm{v} / \mathrm{v})$ for 10 minutes at room temperature. The sections were finally rinsed in methanol-isopropanol, dehydrated, cleared in xylol, and mounted.

Ultrathin sections were contrasted with uranyl acetate and lead citrate for electron microscopy. Electron photomicrographs were taken with a JOEL (JEM, 1200EX, Japan) transmission electron microscope. 


\section{RESULTS}

Classification of muscle fiber types

Succinate dehydrogenase (SDH) activity in pigeon muscles indicated the highest acivity in the red type fiber of the pectoral muscles, whereas only trace staining was seen in the white type fiber (Fig. 1). Both red and white type fibers showed moderate activity for myosin ATPase following preincubation at $\mathrm{pH} 10.5$ and $\mathrm{pH} 4.6$, but negative staining following preincubation at $\mathrm{pH} 4.2$ (Fig. 2b). These two types of muscle fibers in the pigeon pectoral muscle were categorized as type IIB by the myosin ATPase reaction (Table 1). In the gastrocnemius muscle, the SDH reaction in fibers was classified into two types, intermediate and slight, based on the staining intensity (Fig. 3), and myosin ATPase activity could also be divided into three types (Fig. 4). The type I fiber of the leg muscle showed negative ATPase staining following preincubation at $\mathrm{pH} 10.5$, whereas type IIA displayed strong reaction and IIB fibers displayed moderate staining (Fig. 4).

Morphology of muscle fibers

Semithin sections stained with the modified PPD method clearly revealed two types of muscle fibers. One type is red, characterized by black granules (probably mitochondria) and larger black fat granular bodies. Another type is white, and lacks both black granules and larger black fat granular bodies (Figs. 5 and 6). Electron microscopic observation of the pectoralis major muscle in the pigeon showed that type IIB red muscle fibers have numerous round fat granules located between muscle fibers

TABLE 1. Histochemical profile of myosin ATPase and SDH activity in Pigeon pectoral and leg muscles.

\begin{tabular}{|c|c|c|c|c|c|}
\hline & \multirow{2}{*}{\multicolumn{2}{|c|}{$\begin{array}{l}\text { Pectoral } \\
\text { Type IIB }\end{array}$}} & \multicolumn{3}{|c|}{ Gastrocnemius } \\
\hline & & & \multirow{2}{*}{ Type I } & \multirow{2}{*}{ Type IIA } & \multirow{2}{*}{ Type IIB } \\
\hline & Red & White & & & \\
\hline \multicolumn{6}{|c|}{$\begin{array}{c}\text { ATPase } \\
\text { Preincubation }\end{array}$} \\
\hline 10.7 & 0 & 0 & 0 & 0 & 0 \\
\hline 10.6 & 0 & 0 & 0 & 0 & 0 \\
\hline 10.5 & +2 & +2 & 0 & +3 & +2 \\
\hline 10.4 & +3 & +3 & 0 & +3 & +3 \\
\hline 10.3 & +3 & +3 & +2 & +3 & +3 \\
\hline 4.7 & +3 & +3 & +3 & +3 & +3 \\
\hline 4.6 & +2 & +2 & +3 & 0 & +2 \\
\hline 4.5 & 0 & 0 & +3 & 0 & 0 \\
\hline 4.4 & 0 & 0 & +3 & 0 & 0 \\
\hline 4.3 & 0 & 0 & +3 & 0 & 0 \\
\hline 4.2 & 0 & 0 & +3 & 0 & 0 \\
\hline 4.1 & 0 & 0 & +2 & 0 & 0 \\
\hline $\mathrm{SDH}$ & +3 & +1 & +2 & +1 & +1 \\
\hline
\end{tabular}

0 : negative, +1 : weak staining, +2 moderate staining, +3 : strong staining 
(Fig. 7). Also, fat granular bodies were occasionally noted beneath the sarcolemma. In the pectoral muscle, mitochondria in the type IIB red fibers were interspersed among the fat granules. The type IIB white muscle fibers contained a lower fewer number of fat granules and mitochondria and had a thin Z-line compared with that of the type IIB red fibers (Fig. 8).

The diameters of muscle fiber types

Fiber diameters of the type IIB red fiber in the pectoral muscle were rather small, $26.8 \mu \mathrm{m} \pm 6.3$; while those of the type IIB white fiber were two times larger, $50.9 \mu \mathrm{m} \pm 7.7$. The type IIB white fibers accounted for only $3.4 \%$ of the fibers and were usually located in the periphery of muscle bundles (Figs. 1, 2).

The diameters of muscle fibers in the gastrocnemius muscle were relatively similar between the I, IIA, and IIB types of fibers, and the fiber population was $23.0 \%$ type I, $33.3 \%$ type IIA and $43.6 \%$ type IIB (Figs. 3,4 )

\section{DISCUSSION}

Muscle fiber types in vertebrates, lower animals and birds have been classified by histological structure (2), as physiologically fast or slow (11), by SDH histochemical reaction as $\mathrm{A}, \mathrm{B}$, and $\mathrm{C}$ fibers $(10,18)$, white, intermediate and red fibers $(15,16)$, type I and II fibers (6), and $\alpha, \alpha \beta$, and $\beta$ muscle fibers $(8,9)$. In general the red fibers possessed high oxidative enzyme content, low glycolytic enzyme content and the white fibers a high glycolytic enzyme content. In the present study, the flight muscle of the pigeon, the pectoralis major muscle was composed of numerous red fibers and very few white fibers. However, classification of muscle fibers has been accepted based on the staining for myosin ATPase activity with acid or alkaline pretreatment. Thus, skeletal muscle fibers may now be typed as type I, IIA, IIB, and IIC in terms of metabolic and physilogical significance (1). However, it has been demonstrated that the histochemical demonstration of myosin ATPase is markedly influerced by many factors including the time, temperature, $\mathrm{pH}$, and we also detected muscle fibers at various $\mathrm{pH}$ 's $(0.1 \mathrm{pH}$-steps). Based on this classification system, flight muscle fibers of pigeon pectoral muscle fell into the type IIB fiber (fast-twitch).

Almost all muscle fibers in the pigeon pectoral muscle are rich in mitochondoria, have high SDH activity and contain many fat granules located among the mitochon-

FIG. 1. Cryostat cross-section of pigeon pectoralis muscle incubated for SDH activity $(\times 80)$. All type IIB red fibers show high reaction, while type IIB white fibers (periphery of the muscle bundles) display poor reaction.

FIg. 2a. Cryostat cross-section of pectoral muscle showing myosin ATPase activity at a preincubation of $\mathrm{pH} 10.5(\times 80)$. Both "IIB white" and "IIB red" fibers display high reaction.

FIG. 2b. Serial section of Fig. 2a; myosin ATPase, pH 4.2. Both "IIB white" and "IIB red" fibers display no staining.

FIG. 3. Cryostat cross-section of pigeon gastrocnemius muscle for $\mathrm{SDH}$ reaction $(\times 80)$.

FIG. 4a. Cryostat cross-section of gastrocnemius muscle stained for myosin ATPase follwing preincubation at $\mathrm{pH} 10.5(\times 80)$

FIG. 4b. Serial section of Fig. 4a; myosin ATPase, pH 4.6.

FIg. 4c. Serial section of Fig. 4b; myosin ATPase, $\mathrm{pH} 4.2$.

Type I fibers (I) are not stained in Fig. 4a., but are high reaction in Figs. 4b and 4c.

Type IIA fibers (A) are high stained in Fig. 4a, negative in Figs. $4 \mathrm{~b}$ and 4c.

Type IIB fibers (B) are moderately stained in Figs. $4 a$ and $4 b$, no reaction in Fig. 4c. 


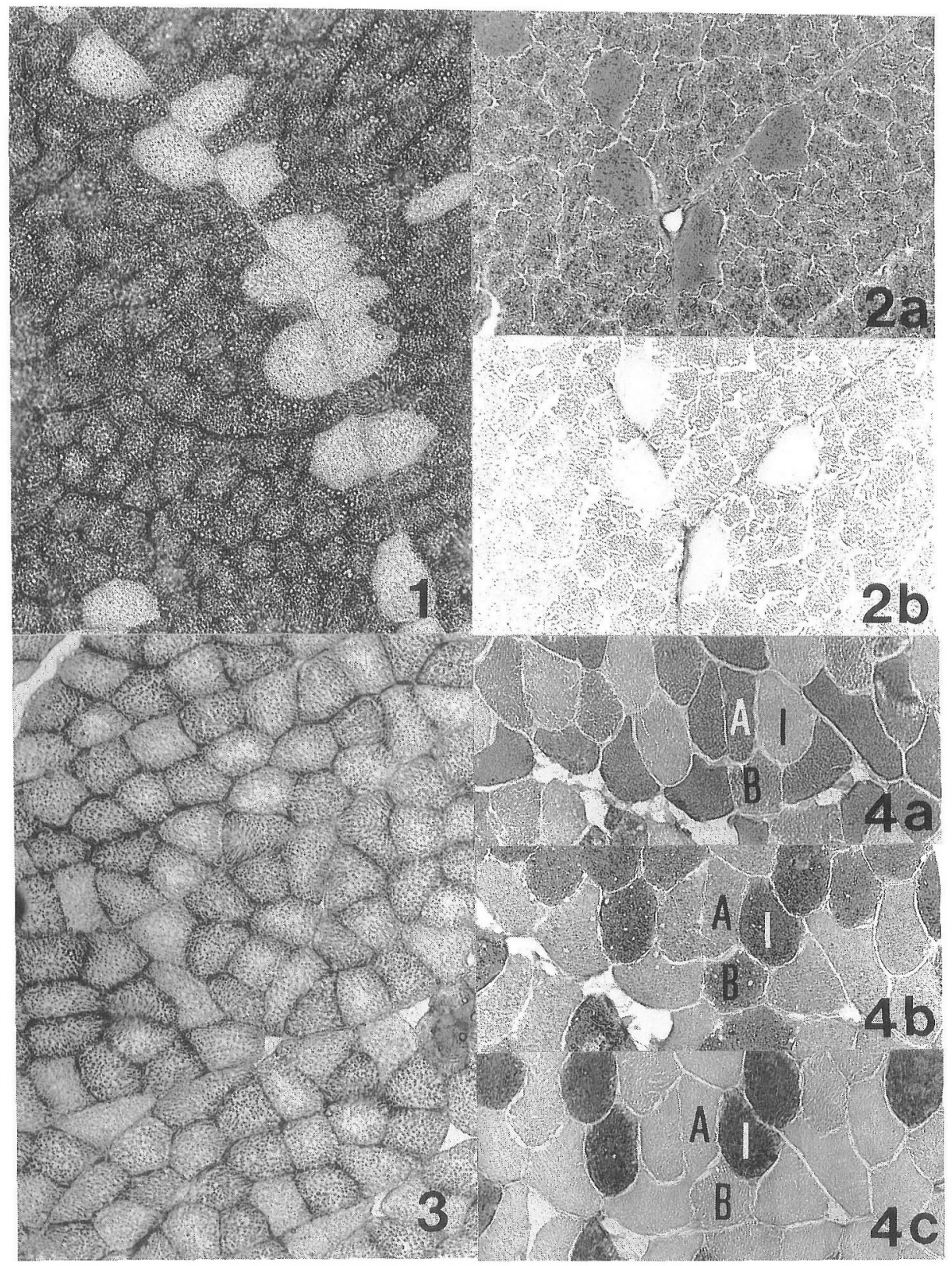




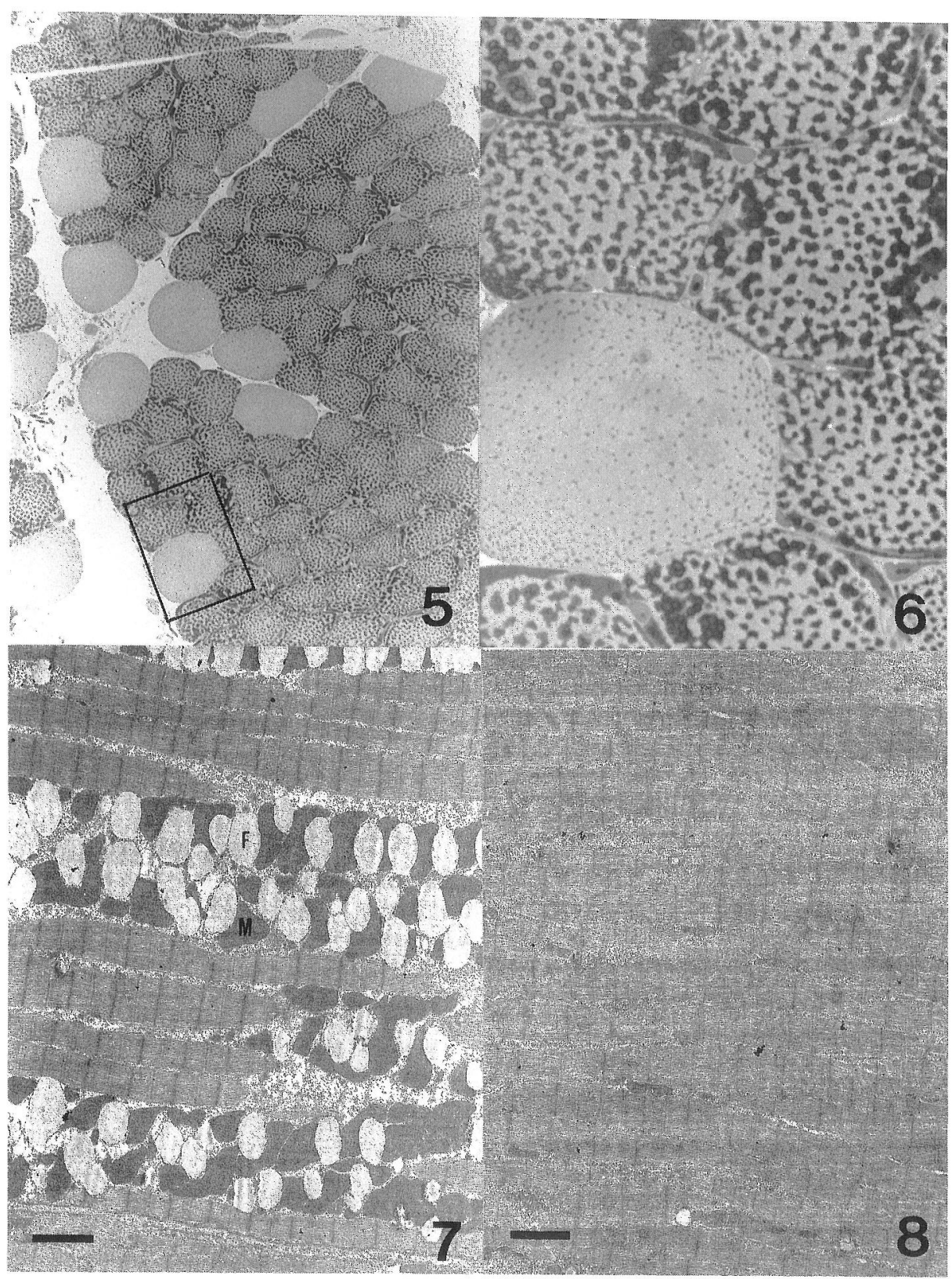

FIG. 5. Semithin cross-section of pigeon pectoralis muscle stained with modified p-phenylendiamine $(\mathrm{PPD})$ method $(\times 80)$. Type IIB red fibers, with small diameter, are darkly stained, Type IIB white are lightly stained

FIG. 6. Enlargement of rectangle in Fig. $5(\times 320)$. A large number of smaller black granules (probably mitochondria) and larger black fat granules in type IIB red fibers.

FIG. 7. Ultrathin longitudinal section of pectoralis muscle $(\times 2,400)$. Bar $=2 \mu \mathrm{m}$. Type IIB red fiber (top) show abundant intermyofibrillar mitochondria (M) and fat droplets $(\mathrm{F})$. Bottom; Type IIB white fiber.

FIG. 8. Ultrathin section type IIB white fiber of the pectoralis $(\times 2,400) . \quad B a r=2 \mu \mathrm{m}$ 
dria; and, as stated above, are mostly type IIB red fibers. These muscle fibers are thus endowed with resistance against fatigue due to long distance flying. The large numbers of fat granules present in the type IIB red fiber probably represent an energy source for intense muscle exercise, but type IIB white fiber is only used in taking off. Similar features of the pigeon pectoral muscle have been reported by Khan (13), who suggested that the red fibers should be categorized as fast-twitch-red or type II red, but not slow-twitch or type I. He also noted that in pigeon pectoral muscle, the existence of high ATPase activity, high fat, and strong SDH activity, and the type of myofibrillar arrangement were meaningful for fast-twitch fatigue resistant or type II fibers. In the present study, measurements of muscle fiber numbers and diameters with a semi-automatic image analyzer showed that almost all muscle fibers of the pigeon pectoral muscle consisted of the type IIB red fibers with a small diameter. Edman (5) noted that great variations were found in fiber size, activities for oxidative and glycolytic enzymes, and Z-band and M-band structures in the "white" region of chicken breast muscle. Talesara and Goldspink (19) studied histochemically and biochemically myosine ATPase in pectoral, leg and cardiac muscles of several avian species and there are difficulties in classifying fibers of the avian muscles, paticularly of the pectoralis.

On the contrary, in the gastrocnemius muscle with myosin ATPase activity could also be divided into three types (I, IIA and IIB). Futhermore the fat granule content was lower than in the pectoralis major.

Muscle fibers in transverse semithin sections stained with PPD were classified into $\mathrm{M}$, INT and $\mathrm{H}$ types, and this classification of muscle fibers corresponds to the ATPase categorization (12) as follows: the majority of the M-fibers are type I; the $\mathrm{H}$ fibers contains mostly the type IIB but also relatively high amounts of type IIA; few type IIC fibers are mainly of the $\mathrm{M}$ and INT fibers variety. In the present study of pigeon pectoral muscle, fibers could be clearly divided between red type (high oxidative and fat) and white type (low oxidative and fat) in semithin sections of osmiumfixed and epon embedded muscle tissue by the modification of PPD staining.

These findings from two different muscles of the pigeon were evaluated for biological correlation between the histological features of the fibers and their metabolism.

\section{ACKNOWLEGEMENT}

This investigation was supported by MIYATA Research Fund.

\section{REFERENCES}

1. Brooke, M. H. and Kaiser, K. K.: Muscle fiber types: How many and what kind? Arch. Neurol. 23; 369-379, 1970.

2. Denny-Brown, D. E.: The histologic features of striped muscle in relation to its functional activity. Proc. Roy. Soc. B104; 371-411, 1929.

3. Dubowitz, V. and Pearse, A. G. E.: A comparative histochemical study of oxidative enzyme and phosphorylase activity in skeletal muscle. Histochernie 2; 105-117, 1960.

4. Dubowitz, V.: Muscle biopsy: a practical approach. 2ed ed., Bailliere Tindall, London, pp. $56-81,1985$. 
5. Edman, A. C., Lexell, J., Sjostrom, M. and Squire, J. M.: Structural diversity in muscle fibers of chicken breast. Cell Tissue Res. 251; 281-289.

6. Engel, W. K.: The essentiality fo histo and cytochemical studies of skeletal muscle in the investigation of neuromusclar disease. Neurology 12; 778-794, 1962.

7. Gauthier, G. F.: On the relationship of ultrastructural and cytochemical features to color in mammalian skeletal muscle. Z. Zellforsch. 95; 462-482, 1969.

8. Green, H. J., Reichmann, H, and Pette, D.: A comparsion of two ATPase based schemes for histochemical muscle fiber typing in various mammals. Histochemistry 76; 21-31, 1982.

9. Guth, L. and Samaha, F. J.: Procedure for the histchemical demonstratoin of actomyosin ATPase. Exp. Neurol. 28; 365-367, 1970.

10. Hennenman, E. and Olson, C. B.: Relations between structure and function in design of skeletal muscles. J. Neurophysiol. 28; 581-598, 1965.

11. Hess, A.: Structural differences of fast and slow extrafusal muscle fibers and their nerve endings in chickens. J. Physiol. 157; 221-231, 1961.

12. Ingjer, F.: Correlation of individual skeletal muscle fibers from "semithin" sections stained with p-phenylene-diamine and histochemical sections incubated for myofibrillar ATPase. Histochemistry 60; 107-111, 1979.

13. Khan, M. A.: "Red" fibers fo pectoralis major muscle are "Type II Red". Histochemistry 55; 75-79, 1978.

14. Korneliussen, H.: Identification of muscle fiber types in "semithin" sections stained with pPhenylene-diamine. Histochemie 32; 95-98, 1972.

15. Ogata, T.: A histochemical study of the red and white muscle fibers. Part I. Activity of the succioxydase system in muscle fibers. Acta Medica. Okayama 12; 216-227, 1958.

16. Ogata, T. and Mori, M.: Histochemical study of oxidative enzymes in vertebrate muscles. $J$. Histochem. Cytochem. 12; 171-182, 1964.

17. Rosser, B. W. C., Daivis, M. B., Brocklebank, J. R. and George, J. C.: On the histochemical characterization and distribution of fast and slow muscle fibers in certain avian skeletal muscles. Acta histochem. 81; 85-93, 1987.

18. Stein, J. M. and Padykula, H. A.: Histochemical classification of individual of skeletal muscle fibers of the rat. Am. J. Anat. 100; 103-124, 1962.

19. Talesara, G. L. and Goldspink, G.: A combined histochemical and biochemical study of myofibrillar ATPase in pectoral, leg and cardiac muscle several species of bird. Histochem. J. $10 ; 695-710,1978$. 